\title{
Usefulness of cholangioscopic-guided mapping biopsy using SpyGlass DS for preoperative evaluation of extrahepatic cholangiocarcinoma: a pilot study
}

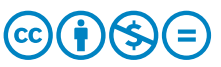

\author{
Authors \\ Takahisa Ogawa, Kei Ito, Shinsuke Koshita, Yoshihide Kanno, Kaori Masu, Hiroaki Kusunose, Toshitaka Sakai, \\ Toji Murabayashi, Sho Hasegawa, Yutaka Noda
}

Institution

Department of Gastroenterology, Sendai City Medical Center, Sendai, Japan

submitted 10.2.2017

accepted after revision 22.6.2017

Bibliography

DOI https://doi.org/10.1055/s-0043-117949 |

Endoscopy International Open 2018; 06: E199-E204

(c) Georg Thieme Verlag KG Stuttgart · New York ISSN 2364-3722

\section{Corresponding author}

Takahisa Ogawa, Sendai City Medical Center, 5-22-1, Tsurugaya, Miyagino-ku, Sendai 983-0824, Japan

Fax: +81-22-252-9431

t-ogawa@openhp.or.jp

\section{ABSTRACT}

Background and study aims Evaluation of longitudinal tumor extent is indispensable for curative surgical treatment of extrahepatic cholangiocarcinoma. The aim of this study was to evaluate the usefulness and feasibility of cholangioscopic-guided mapping biopsy using a newly developed peroral digital cholangioscope, SpyGlass DS (SpyDS), for preoperative evaluation of extrahepatic cholangiocarcinoma.
Patients and methods Thirteen patients (mean age, 75 years; male 10, female 3 ) with extrahepatic cholangiocarcinoma who underwent cholangioscopic-guided mapping biopsy using SpyDS for preoperative evaluation were included in this study. Successful cholangioscopic-guided mapping biopsy was defined as the acquisition of specimens sufficient for histopathological diagnosis.

Results The mean number of biopsies was 5 per patient. The overall success rate for cholangioscopic-guided mapping biopsy was $88 \%(59 / 67)$. The success rate for cholangioscopic-guided mapping biopsy from the confluence of the right and left hepatic ducts was $89 \%$, that from the B4 confluence was $93 \%$, that from the confluence of the right anterior and right posterior segmental ducts was $86 \%$, that from the intrapancreatic common bile duct was $67 \%$, and that from the main lesion was $100 \%$. The overall diagnostic accuracy of longitudinal tumor extent at the hepatic side, the duodenal side and overall by cholangioscopic findings and mapping biopsy, was $88 \%$ (7/8), $88 \%(7 / 8)$ and $88 \%(7 / 8)$, respectively. Assessment according to location of the main lesion revealed that diagnostic accuracy in the patients with distal bile duct carcinoma was $100 \%$ (5/5) and that in patients with perihilar bile duct carcinoma was $66 \%$ (2/3). Complications after the procedure did not occur in any patients.

Conclusions Cholangioscopic-guided mapping biopsy using SpyDS is thought to be feasible for preoperative evaluation of extrahepatic cholangiocarcinoma.

\section{Introduction}

Extrahepatic cholangiocarcinoma frequently extends longitudinally along the bile duct. Preoperative evaluation of tumor extent is essential for estimating operability and for determining the most appropriate resection line. Endoscopic transpapillary mapping biopsy has been reported to be useful for preoperative evaluation of the extent of extrahepatic cholangiocarcinoma [1-4]. However, guiding biopsy forceps to the target sites of the bile duct is quite difficult due to its poor maneuverability.
Therefore, adequate endoscopic transpapillary mapping biopsy remains challenging.

A newly developed peroral digital cholangioscope, SpyGlass DS Direct Visualization System (SpyDS) (Boston Scientific Co., Natick, MA, USA), is characterized by excellent maneuverability with 4 tip directions, good image quality, and easy irrigation with dedicated channels. Recently, the diagnostic and therapeutic feasibility of SpyDS has been reported in a few studies [5-7]. However, there have been no studies on cholangioscopic-guided mapping biopsy using SpyDS for preoperative evaluation of extrahepatic cholangiocarcinoma. 
The aim of this pilot study was to evaluate the usefulness and feasibility of cholangioscopic-guided mapping biopsy using SpyDS for preoperative evaluation of extrahepatic cholangiocarcinoma.

\section{Patients and methods}

\section{Patients}

Between October 2015 and September 2016, 23 patients were diagnosed with extrahepatic cholangiocarcinoma at our institution. Of them, patients who did not meet the following exclusion criteria underwent cholangioscopic-guided mapping biopsy using SpyDS for preoperative evaluation and were enrolled in this study. Exclusion criteria were as follows: (1) refusal of surgical treatment; (2) definite inoperable factors such as distal metastases and peritoneal dissemination detected by multi-detector row computed tomography (MDCT); (3) tumors that definitely involved both the B4 confluence and the confluence of the right anterior and right posterior segmental ducts on the basis of findings of MDCT, magnetic resonance cholangiopancreatography (MRCP), or endoscopic ultrasonography (EUS); and (4) poor general condition (performance status 3 or 4). Eventually, 13 patients were included in this study. Written informed consent was obtained from all patients before examinations. This study was approved by the institutional review board of Sendai City Medical Center (approval number, 2016-0029).

\section{Specifications for SpyGlass DS direct visualization system}

SpyDS is composed of SpyScope DS and SpyGlass DS Digital Controller. SpyDS is characterized by a simplified set-up (setup time of under 5 minutes), automatic white balance and focus, excellent maneuverability with four tip directions, good image quality due to video scope, and easy irrigation with dedicated channels. Specifications for SpyDS are as follows: field of view, $120^{\circ}$; view depth, $1.5 \mathrm{~mm}$; compatible scope channel, $3.7 \mathrm{~mm}$; catheter outer diameter, $3.46 \mathrm{~mm}$ (tip), $3.56 \mathrm{~mm}$ (catheter); scope length, $2140 \mathrm{~mm}$; channel diameter, $1.2 \mathrm{~mm}$.

\section{Procedure for cholangioscopic-guided mapping biopsy}

All patients had undergone multi-detector row computed tomography (MDCT) to evaluate the location of the main lesion and operability before cholangioscopy. Endoscopic retrograde cholangiography (ERC) and endoscopic sphincterotomy were performed and then the SpyDS was inserted into the bile duct, along a 0.025 -inch guidewire through the working channel of a therapeutic duodenoscope (TJF-260V, Olympus Co., Tokyo, Japan). If it was difficult to advance the SpyDS to the hepatic side due to severe stricture, dilation using a tapered catheter or dilation balloon was considered. After irrigation of the bile duct with saline, longitudinal tumor extent was evaluated on the basis of observational findings with SpyDS. Findings of longitudinal tumor extent were defined as irregular fine granular or fishegg-like mucosa that continuously spread from the main lesion [2-4]. Next, cholangioscopic-guided mapping biopsy was per- formed using SpyBite Biopsy Forceps (Boston Scientific Co., Natick, MA, USA) ( Fig. 1, \Video 1). Surgical strategy differed according to the location of the main lesion. For instance, if a main lesion is located in the distal bile duct, conceivable surgical procedure is pancreatoduodenectomy (PD) or hepatectomy with PD. On the other hand, if the main lesion is located the perihilar bile duct, conceivable surgical procedure is choledochectomy, choledochectomy with hepatectomy or hepatectomy with PD. Therefore, bile duct sites where mapping biopsy was attempted were determined in consideration of the resection line for a conceivable surgical procedure. Identification of the location of the bile duct during SpyDS was confirmed by correspondence with cholangiography which had been performed before SpyDS with a guidewire that was inserted thorough the Spyscope channel under fluoroscopic guidance.

Outcome measures for this study were the success rate of cholangioscopic-guided mapping biopsy, diagnostic accuracy of longitudinal tumor extent using SpyDS (cholangioscopic findings and cholangioscopic-guided mapping biopsy), and complications after the procedure. Success with cholangioscopic-guided mapping biopsy was defined as acquisition of specimens sufficient for histopathological diagnosis. Specimens were considered sufficient for histopathological diagnosis if they included epithelium sufficient for judgment as to benign or malignant status. Longitudinal tumor extent was confirmed by histological examination of the resected specimens. Diagnostic accuracy of longitudinal tumor extent using SpyDS was defined as corresponding with the tumor extent confirmed by histological examination of resected specimens. Cases that ultimately became inoperable were excluded from assessment of diagnostic accuracy of longitudinal tumor extent. Complications were according to consensus criteria [8].

\section{Results}

Thirteen patients (mean age of 75 years, 10 males, 3 females) were enrolled in this study. The location of the main lesion was the perihilar bile duct in 7 patients (Bismuth type I in 3, type II in 1, type IIla in 3) and the distal bile duct in 6 .

Cholangioscopic-guided mapping biopsy was attempted from the confluence of the right and left hepatic ducts in 10 patients, the B4 confluence in 7, the confluence of the right anterior and right posterior segmental ducts in 4 , the intrapancreatic common bile duct in 4 and the main lesion in 6 (the perihilar bile duct in 5 and the distal bile duct in 1). The mean number of biopsies was 5 per patient (range, $3-10$ ). Insertion of the SpyDS to the hepatic side beyond the biliary stricture and acquisition of specimens from target sites macroscopically were successful in all patients. However, $12 \%$ (8/67) of specimens were insufficient for histological examination. In 1 case, dilation of the stricture using a $10 \mathrm{Fr}$ tapered catheter was needed before insertion of the SpyDS. In no cases was dilation using a dilation balloon necessary. The overall success rate for cholangioscopic-guided mapping biopsy was $88 \%$ (59/67). The success rate for cholangioscopic-guided mapping biopsy from the confluence of the right and left hepatic ducts was $89 \%$, that from the B4 confluence was $93 \%$, that from the confluence 

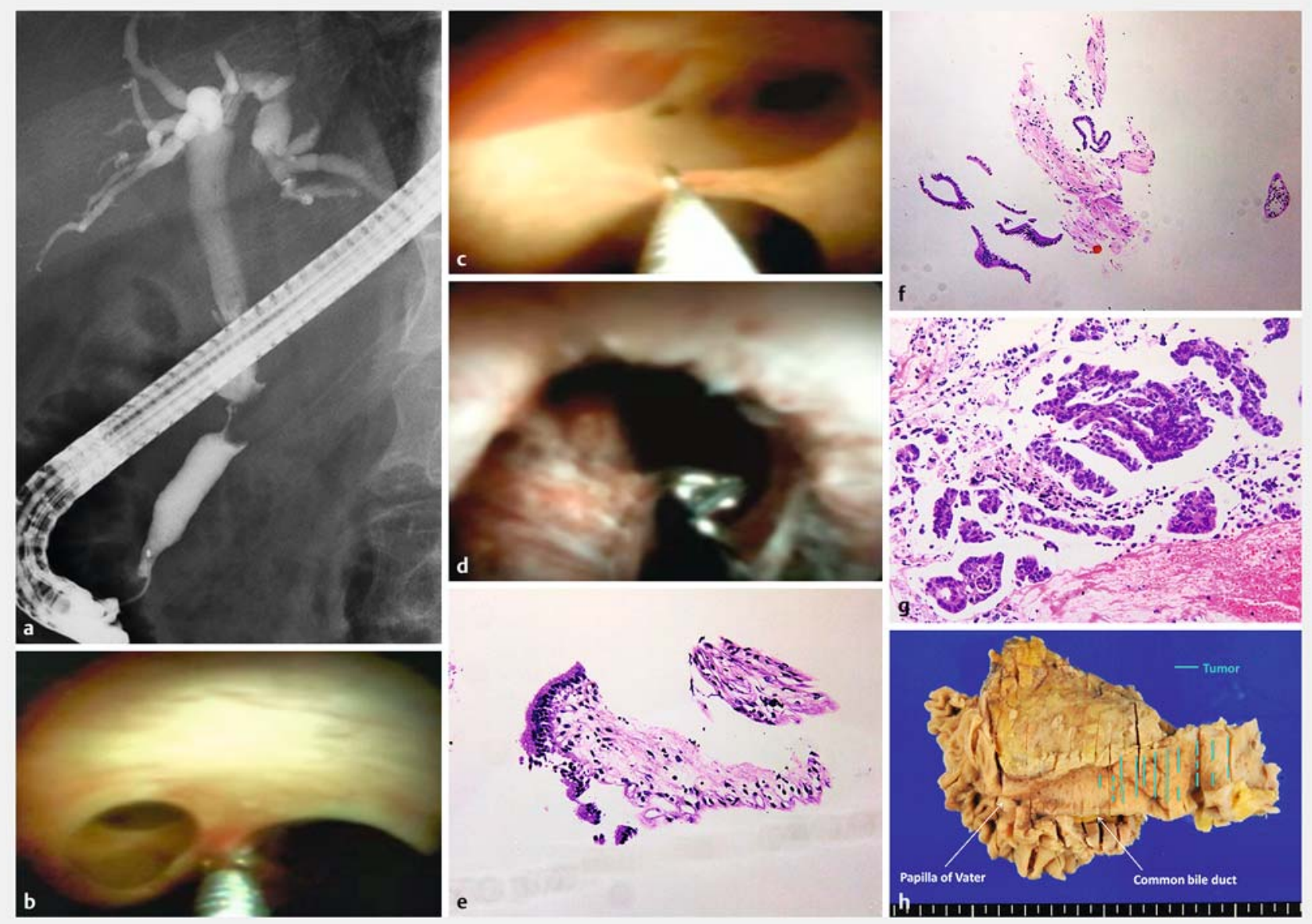

- Fig. 1 a A case of distal cholangiocarcinoma. ERC demonstrated a distal bile duct stricture. b Cholangioscopic-guided mapping biopsy was performed from the B4 confluence, $\mathbf{c}$ the confluence of the anterior and posterior segmental ducts, $\mathbf{d}$ and the main lesion. $\mathbf{e}$ Histopathological examination of biopsy specimens revealed that the B4 confluence and $\mathbf{f}$ the confluence of the anterior and posterior segmental ducts had nonneoplastic mucosa and that $\mathbf{g}$ the main lesion had adenocarcinoma. $\mathbf{h}$ Mapping of the resected specimen.

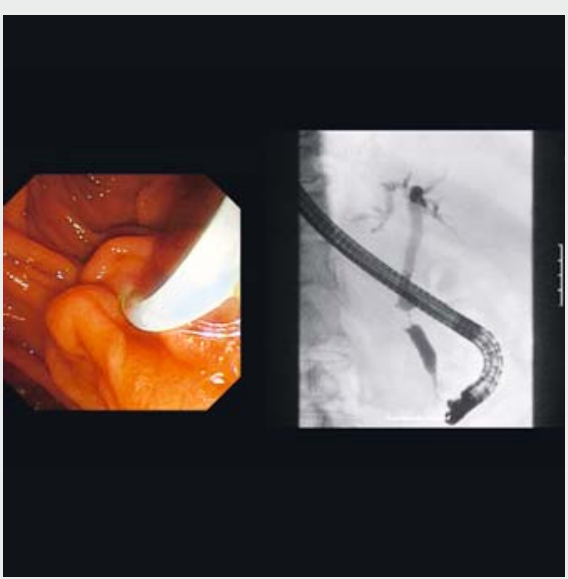

Video 1 The case of distal cholangiocarcinoma in $>$ Fig. 1. of the right anterior and right posterior segmental ducts was $86 \%$, that from the intrapancreatic common bile duct was $67 \%$, and that from the main lesion was $100 \%$ ( $\triangleright$ Table 1 ).

In consideration of results of the preoperative examinations including SpyDS and tolerance to surgery of each patient, 5 patients were estimated as being inoperable. The remaining 8 patients underwent surgery and histopathological examination of resected specimen. These patients were evaluated as to the diagnostic accuracy of longitudinal tumor extent. With only cholangioscopic findings, the diagnostic accuracy of longitudinal tumor extent at hepatic side, duodenal side and overall was $75 \%(6 / 8), 88 \%(7 / 8)$ and $75 \%(6 / 8)$, respectively. With the addition of cholangioscopic-guided mapping biopsy, the diagnostic accuracy at hepatic side and overall increased to $88 \%(7 / 8)$ ( $\vee$ Table 2). Assessment according to location of the main lesion revealed that the diagnostic accuracy in patients with distal bile duct carcinoma was $100 \%(5 / 5)$ and that in patients with perihilar bile duct carcinoma it was $66 \%$ (2/3) ( Table 2). The case misdiagnosed by SpyDS was perihilar bile duct carcinoma (Bismuth type IIla). The tumor involved the confluence of the right anterior and right posterior segmental ducts by several 
- Table 1 Success rate for cholangioscopic-guided mapping biopsy.

Successful cholangioscopic mapping biopsy

Overall $59 / 67(88 \%)$

Location of biopsy

- Confluence of the right and left hepatic ducts $24 / 27(89 \%)$

- B4 confluence $13 / 14(93 \%)$

- Confluence of the anterior and posterior segmental ducts $6 / 7(86 \%)$

- Intrapancreatic common bile duct $6 / 9(67 \%)$

- Main lesion $10 / 10(100 \%)$

modalities performed before SpyDS. Hence, right hepatic lobectomy with caudate lobectomy and choledochectomy would be thought to be necessary at minimum. The B4 confluence and intrapancreatic bile duct were judged to be intact by both visual assessment and mapping biopsy using SpyDS. Therefore, right hepatic lobectomy with caudate lobectomy and choledochectomy was carried out. However, an intramural spreading tumor with non-neoplastic mucosa was revealed at both hepatic and duodenal sides of margins in the histological examination of the resected specimen ( $\bullet$ Fig. 2 ).

No complications after the procedure occurred in any patients.

\section{Discussion}

Evaluation of longitudinal tumor extent is indispensable for curative surgical treatment of extrahepatic cholangiocarcinoma. Several imaging modalities such as ERC, MDCT, intraductal ultrasonography (IDUS), magnetic resonance cholangiopancreatography (MRCP) and peroral cholangioscopy (POCS) have been reported to be useful for preoperative evaluation of extrahepatic cholangiocarcinoma [1-4,9]. Endoscopic transpapillary mapping biopsy combined with such imaging modalities can contribute to improved accuracy as to the longitudinal tumor extent [1-3]. Generally, endoscopic transpapillary mapping biopsy is performed under fluoroscopic or cholangioscopic guidance. Cholangioscopic guidance allows biopsy under direct visualization of bile duct mucosa. It enables a more targe- ted biopsy as well as evaluation on the basis of cholangioscopic findings [2-4]. This is its greatest advantage over fluoroscopic guidance. However, a conventional digital cholangioscope has poor maneuverability with only 2 tip directions. Therefore, it is sometimes difficult to approach targeted bile ducts, especially the intrahepatic bile ducts such as the B4 confluence and the confluence of the anterior and posterior segmental ducts [1, 4]. On the other hand, the SpyDS has excellent maneuverability with 4 tip directions, which enables the endoscopist to easily approach even intrahepatic bile ducts. In the current study, approaching targeted sites, including intrahepatic bile ducts, was successful in all patients.

The outer diameter of a cholangioscope, including SpyDS, is over $10 \mathrm{Fr}$. Therefore, a cholangioscope sometimes cannot be advanced through a severe stricture. Previous study on a conventional digital cholangioscope reported that it was impossible to advance through stricture in $30.2 \%$ of patients [4]. However, in the current study, advancing the scopes through the stricture was successful in all cases although stricture dilation was needed in 1 patient. Possible reasons for this result are as follows: (1) there were no severe strictures in this study cases; and (2) insertability of SpyDS is good. Further studies are required to clarify the insertability of SpyDS because the number of patients in this study was small.

Usable biopsy forceps in cholangioscopic-guided biopsy are small because the channel of the cholangioscope is narrow. Hence, one of the concerns about cholangioscopic-guided biopsy is the size limitation for specimens compared with fluoroscopic-guided biopsy [4]. Nishikawa et al. reported that $58.8 \%$ and $76.0 \%$ of cholangioscopic biopsy specimens from secondary biliary radicles and the intrapancreatic bile duct, respectively, were insufficient for histological evaluation [4]. In the current study, although specimen acquisition from target sites macroscopically was successful in all patients, $12 \%$ of specimens were insufficient for histological examination. In particular, the success rate for mapping biopsy from intrapancreatic bile duct was lower than that from other sites. The reason for this result is presumed to be that biopsy from the intrapancreatic duct is prone to be difficult due to the tangential direction. Although the proportion of insufficient specimens in our study was lower than that in a previous report [4], biopsy forceps should be improved so as to obtain bigger specimens.

In this study, diagnostic accuracy of longitudinal tumor extent with cholangioscopic findings and cholangioscopic-guided mapping biopsy was $88 \%$. In a prospective multicenter study on preoperative evaluation of cholangiocarcinoma using a conven-

- Table 2 Diagnostic accuracy of longitudinal tumor extent.

\begin{tabular}{|l|l|l|l|l|l|l|}
\hline & \multicolumn{2}{|c|}{ Cholangioscopic findings only } & \multicolumn{2}{l|}{ Cholangioscopic findings plus mapping biopsy } \\
\hline Location of the main lesion & Overall & Hepatic side & Duodenal side & Overall & Hepatic side & Duodenal side \\
\hline Perihilar bile duct & $1 / 3(33 \%)$ & $1 / 3(33 \%)$ & $2 / 3(66 \%)$ & $2 / 3(66 \%)$ & $2 / 3(66 \%)$ \\
\hline Distal bile duct & $5 / 5(100 \%)$ & $5 / 5(100 \%)$ & $5 / 5(100 \%)$ & $5 / 5(100 \%)$ & $5 / 5(100 \%)$ & $5 / 5(100 \%)$ \\
\hline Overall & $\mathbf{6 / 8}(\mathbf{7 5} \%)$ & $\mathbf{6 / 8 ( 7 5 \% )}$ & $\mathbf{7 / 8 ( 8 8 \% )}$ & $\mathbf{7 / 8 ( 8 8 \% )}$ & $\mathbf{7 / 8 ( 8 8 \% )}$ \\
\hline
\end{tabular}



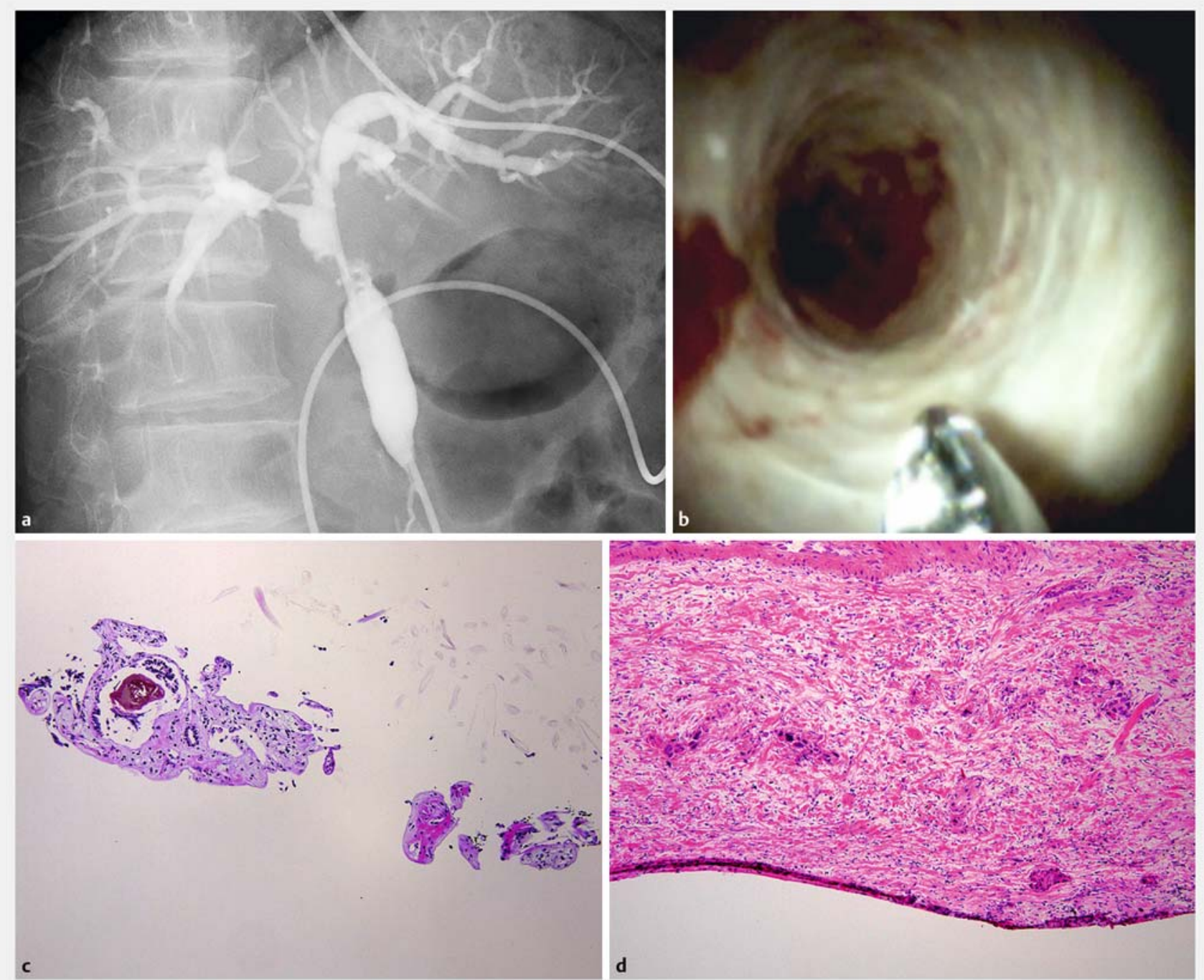

- Fig. 2 A case misdiagnosed by SpyDS. a The main lesion was located in the perihilar bile duct and Bismuth classification was type IIla by. b Both the visual assessment and $\mathbf{c}$ mapping biopsy using SpyDS did not detect tumor extent at the B4 confluence and the intrapancreatic bile duct. d However, an intramural spreading tumor with non-neoplastic mucosa was revealed at both hepatic and duodenal sides of margins.

tional video cholangioscope, the accuracy rate of longitudinal tumor extent with ERC with POCS plus mapping biopsy was reported to be $92.9 \%$ [3], comparable to our study result. The longitudinal extent of extrahepatic cholangiocarcinoma includes two forms, i.e., mucosal extent and intramural extent. The cases which could not be diagnosed in our study had only intramural tumor extent with non-neoplastic mucosa. To diagnose such cases accurately, biopsy of deep tissue is necessary. However, it is assumed to be difficult with cholangioscopicguided mapping biopsy because the biopsy forceps in current use are small.

Our study has several limitations. First, it was retrospective and enrolled a small number of patients. Second, no comparison with fluoroscopic mapping biopsy was made. Because useable biopsy forceps in fluoroscopic-guided biopsy are larger than those in cholangioscopic-guided mapping biopsy, the size of biopsy specimens under fluoroscopic guidance can be expected to be greater than in the case of cholangioscopic guidance. Further study comparing cholangioscopic-guided mapping biopsy with fluoroscopic mapping biopsy is needed to evaluate the superiority of cholangioscopic-guided mapping biopsy. Third, the accuracies of other imaging modalities were not evaluated in this study. Therefore, the degree of improvement in accuracy by use of cholangioscopic-guided mapping biopsy is unclear. Fourth, comparison with the first-generation SpyGlass was not made in this study. According to literature searches using PubMed, there have been no studies on the use of first-generation of SpyGlass for preoperative evaluation of longitudinal tumor extent of extrahepatic cholangiocarcinoma. Although the maneuverability of first-generation Spyclass is thought to be comparable to that for SpyDS, image quality of first-generation SpyGlass is very poor due to the fiberscope. For evaluation of longitudinal tumor extent of extrahepatic cholangiocarcinoma using cholangioscopy, both maneuverabil- 
ity and image quality are very important. Therefore, SpyDS is thought to be superior to first-generation SpyGlass in preoperative evaluation of extrahepatic cholangiocarcinoma.

\section{Conclusion}

In this pilot study, mapping biopsy using SpyDS was found to be useful and feasible for preoperative evaluation of extrahepatic cholangiocarcinoma. Further study is needed to establish evidence about the usefulness of cholangioscopic-guided mapping biopsy using SpyDS.

Competing interests

None

\section{References}

[1] Noda Y, Fujita N, Kobayashi G et al. Intraductal ultrasonography before biliary drainage and transpapillary biopsy in assessment of the longitudinal extent of bile duct cancer. Dig Endosc 2008; 20: 73-78

[2] Kawakami H, Kuwatani M, Etoh $\mathrm{K}$ et al. Endoscopic retrograde cholangiography versus peroral cholangioscopy to evaluate intraepithelial tumor spread in biliary cancer. Endoscopy 2009; 41: 959-964
[3] Osanai M, Itoi T, Igarashi Y et al. Peroral video cholangioscopy to evaluate indeterminate bile duct lesions and preoperative mucosal cancerous extension: a prospective multicenter study. Endoscopy 2013; 45: 635-641

[4] Nishikawa T, Tsuyuguchi T, Sakai Y et al. Preoperative assessment of longitudinal extension of cholangiocarcinoma with peroral videocholangioscopy: A prospective study. Dig Endosc 2014; 26: 450 - 457

[5] Tanaka R, Itoi T, Honjo M et al. New digital cholangiopancreatoscopy for diagnosis and therapy of pancreaticobiliary deseases (with videos). J Hepatobiliary Pancreat Sci 2016; 23: 220-226

[6] Navaneethan U, Hasan MK, Kommaraju K et al. Digital, single-operator cholangiopancreatoscopy in the diagnosis and management of pancreatobiliary disorders: a multicenter clinical experience (with video). Gastrointest Endosc 2016; 84: 649-655

[7] Varadarajulu S, Bang JY, Hasan MK et al. Improving the diagnosistic yield of single-operator cholangioscopy-guided biopsy of indeterminate biliary strictures: ROSE to the rescue? (with video) Gastrointest Endosc 2016; 84: 681-687

[8] Cotton PB, Lehman G, Vennes J et al. Endoscopic sphincterotomy complications and their management: an attempt at consensus. Gastrointest Endosc 1991; 37: 83 - 93

[9] Park HS, Lee JM, Choi JY et al. Preoperative evaluation of bile duct cancer: MRI combined with MR cholangiopancreatography versus MDCT with direct cholangiography. AJR Am Roentgenol 2008; 190: $396-405$ 